\title{
A method for performing fully quantum mechanical simulations of gated silicon quantum wire structures
}

\author{
R. Akis · D.K. Ferry • M.J. Gilbert
}

Published online: 17 July 2009

(C) Springer Science+Business Media LLC 2009

\begin{abstract}
As transistors get smaller, fully quantum mechanical treatments are required to properly simulate them. Most quantum approaches treat the transport as ballistic, ignoring the scattering that is known to occur in such devices. Here, we review the method we have developed for performing fully quantum mechanical simulations of nanowire transistor devices which incorporates scattering through a real-space self-energy, starting with the assumption that the interactions are weak. The method we have developed is applied to investigate the ballistic to diffusive crossover in a silicon nanowire transistor device.
\end{abstract}

Keywords Quantum transport · Phonon scattering · MOSFET

\section{Introduction}

The Metal-Oxide-Semiconductor Field-Effect Transistor (MOSFET) has been the workhorse of the semiconductor industry for many years, with progress in size and speed following a well-known scaling relationship [1]. However, as devices get smaller and smaller, quantum mechanical effects become more significant, and one eventually expects

R. Akis $(\bowtie) \cdot$ D.K. Ferry

Department of Electrical Engineering and Center for Solid State Electronics Research, Arizona State University, Tempe,

AZ 85287-5706, USA

e-mail: richard.akis@asu.edu

M.J. Gilbert

Department of Electrical and Computer Engineering, University of Illinois at Urbana-Champaign, Urbana, IL 61801, USA a breakdown of the simple scaling behavior. Correspondingly, the traditional semi-classical tools of device simulation are fast becoming limited. There have been efforts to expand such methods as Monte Carlo and drift-diffusion to incorporate quantum effects $[2,3]$ via an effective quantum potential $[4,5]$ mainly due to quick and easy results when compared to the computational resources devoted to run some current software. This effort has been most notable in Monte Carlo where the effective potential has found some success in predicting some of the quantum phenomena arising in next generation devices [2], such as charge setback from the gate. However, the effective potential and other quantum corrective tools cannot account for some physical phenomena, such as tunneling [6], which will become much more important as the channel length decreases.

There have been many suggestions for different quantum methods to model these devices including simple analytical models [7, 8] Green's function approaches [9-11] coupled Schrodinger approaches [12, 13], and Pauli master equation approaches [14]. However, in each of these methods, the length and the depth are modeled rigorously, while the third dimension is usually included through the assumption that there is no interesting physics to capture in this dimension. Therefore, the third dimension is usually treated using a basis expansion which is then included in the Hamiltonian. It is assumed also that the mode does not change shape as it propagates from the source of the device to the drain of the device. Other simulation proposals have simply assumed that only one subband in the orthogonal direction is occupied, therefore making higher-dimensional transport considerations unnecessary. This is certainly not a valid assumption. While it is true that the mode will not change shape as it propagates from the source to the drain, it is important to consider the fact that this mode will couple to other modes, which is required to treat some of the interesting physics. In 
the source of the device, the modes that are excited are three dimensional (3D) in nature. These modes are then propagated from the 3D section of the source to the channel. The excitation of different modes changes as one approaches the drain, due to the large source-drain bias. Moreover, as the doping and the Fermi level in short channel MOSFETs increases, we can no longer assume that there is only one occupied subband even at the source.

Another difficulty that quantum simulators typically encounter is how to properly account for dissipation. There have been many different approaches that have been developed to attempt to deal with this issue. Statistical approaches introduce random phase fluctuations into the simulations $[15,16]$. The major drawback of such an approach is that a large sample space is required over which to average, and this entails a great many runs to have any valuable results. Another method is to add an imaginary term to the Hamiltonian which represents the phase breaking time of the electron in the system under consideration $[17,18]$. While this approach seems reasonable for a system that is in equilibrium, it is not clear that it is applicable when the system is driven out of equilibrium. The imaginary term is constant throughout the device, and therefore fails to consider the inhomogeneous density in the out of equilibrium system. This approach has also been questioned as not conserving current [19], but this fails to properly consider the entire dissipative current [20]. Dissipation may also be included through the use of Büttiker probes [21, 22]. While this approach is an improvement over the use of a phasebreaking related term, in that it is current conserving, it suffers from the fact that an additional loop must be included to insure that the probes do not change the number of electrons in the system, nor does it account for the spatial inhomogeneity of the density and the scattering. Moreover, a fitting parameter must be used to calibrate the probes to the proper low field mobility. A relaxation time approximation has also been used in approaches utilizing either the density matrix [23] or the Wigner function [24].

In an effort to address these difficulties, we have developed a more complete simulation method that is fully $3 \mathrm{D}$ and accounts for dissipation in a manner which preserves current conservation and does not require fitting parameters, while at the same time is not as computationally expensive as some other approaches [25-28]. Our simulator uses recursive scattering matrices to determine the current flowing through a given device. During a simulation, one translates from one transverse slice to the next, which happens to be equivalent to solving a local Dyson's equation with each slice Hamiltonian. This means that we can easily modify this Hamiltonian by the direct inclusion of a slice self-energy as well as a self-energy coupling between the slices where that is appropriate. This self-energy term is how we account for dissipation within a device, and we can accurately account for such effects as phonon scattering. Phonon scattering in one and two-dimensional systems (quantum wires and quantum wells, respectively) is usually treated as transitions between transverse modes [29, 30]. Emberly and Kirczenow [31] have introduced inelastic scattering in a recursive wave function approach and Lake and Datta have done so for the non-equilibrium Green's function [32]. But these approaches did not make full use of the computation in real space that is the heart of such recursive approaches, while our method does.

In this paper, we review the technique we have developed for doing fully 3D efficient quantum mechanical transport simulations, specifically on how it can be applied silicon quantum wire structures. In Sect. 2, the recursive scattering matrix method we employ to calculate the transport in quantum devices is described. In Sect. 3, we show how one can include separable scattering mechanisms into such simulations in a site-representation self-energy. How one accounts for acoustic deformation potential scattering and intervalley scattering with both $f$ and $g$ type processes are shown in detail. In Sect. 4, we show an application of the technique that we have developed. We use our simulator to study an SOI quantum wire, tri-gate MOSFET, examining the effect that changing the wire cross-section and length has on the resistance, in order to understand the conditions under which 1D transport and ballisticity are maintained. As we shall show, including electron-phonon scattering produces significant deviations from the ballistic results right down to the length for which the wire can be considered a true waveguide.

\section{The recursive scattering matrix method for quantum transport}

In the case of silicon, there are six equivalent ellipsoids that make up the conduction band. The Schrödinger equation for the wave function contribution from valley $i$ is given by:

$$
\begin{aligned}
& \frac{-\hbar^{2}}{2}\left(\frac{1}{m_{x, i}^{*}} \frac{d^{2}}{d x^{2}}+\frac{1}{m_{y, i}^{*}} \frac{d^{2}}{d y^{2}}+\frac{1}{m_{z, i}^{*}} \frac{d^{2}}{d z^{2}}\right) \Psi^{(i)} \\
& +V(x, y, z) \Psi^{(i)}=E \Psi^{(i)} .
\end{aligned}
$$

Here, it is assumed that the effective masses are constant, in order to simplify the equations (to generalize this to nonparabolic bands, the reciprocal mass would enter between the partial derivatives). The values of the effective masses that enter into (1) depend on how one chooses to orient the device with respect to the crystal axes, which is why the valley index is included in them in (1). A very simple schematic of a silicon device that is examined later in this paper is shown in Fig. 1. For the results shown later, the crystal orientation 
(001)

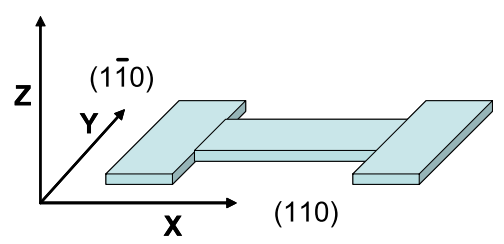

Fig. 1 Simple schematic showing an SOI MOSFET and its orientation with respect to the crystal axes of silicon

of the channel is set so that the current will flow along the $\langle 110\rangle$ direction, so the $x$ axis shown here is parallel to that, while the $z$ axis shown here lines up with $\langle 001\rangle$ direction.

The transport problem is actually solved onto a finite difference grid with uniform spacing $a$, with $x=s a, y=k a$ and $z=\eta a$, where $s, k$ and $\eta$ are integers. Thus, the derivatives in (1) are replaced with finite differences, and the Hamiltonian maps onto a tight-binding model

$$
\begin{aligned}
& -t_{x}^{(i)}\left(\psi_{s+1, k, \eta}^{(i)}+\psi_{s-1, k, \eta}^{(i)}\right)-t_{y}^{(i)}\left(\psi_{s, k+1, \eta}^{(i)}+\psi_{s, k-1, \eta}^{(i)}\right) \\
& -\quad t_{z}^{(i)}\left(\psi_{s, k, \eta+1}^{(i)}+\psi_{s, k, \eta-1}^{(i)}\right) \\
& \quad+\left(V_{s, k, \eta}+2 t_{z}^{(i)}+2 t_{z}^{(i)}+2 t_{z}^{(i)}\right) \psi_{s, k, \eta}^{(i)}=E \psi_{s, k, \eta}^{(i)}
\end{aligned}
$$

with hopping energies given by

$$
\begin{aligned}
t_{x}^{(i)} & =\frac{\hbar^{2}}{2 m_{x, i}^{*} a^{2}}, \quad t_{y}^{(i)}=\frac{\hbar^{2}}{2 m_{y, i}^{*} a^{2}}, \\
t_{z}^{(i)} & =\frac{\hbar^{2}}{2 m_{z, i}^{*} a^{2}} .
\end{aligned}
$$

Given the tight-binding form, an artificial band structure is created. The band along each direction has a cosinusoidal variation with momentum eigenvalue, with the total width of this band being

$W=2 t_{z}^{(i)}+2 t_{z}^{(i)}+2 t_{z}^{(i)}$.

To properly approximate the real band behavior, which is quadratic in momentum, we need to keep the Fermi energies of interest near the bottom of the band, below a value where the cosinusoidal variation deviates significantly from the parabolic behavior. For practical purposes, this means that the maximum Fermi energy must be less than the smallest hopping energy, the latter being a function of $a$. With energies of the order of a $1 \mathrm{~V}$, one is required to have $a$ less than $\sim 0.2 \mathrm{~nm}$. That is, we must take the grid size to be comparable to the silicon lattice spacing or smaller.

The discrete Schrödinger equation (2) can be used to obtain transfer matrices that allows one to translate across the structure, which broken down into a series of slices. We follow a procedure first put forward by Usuki et al. [33] and used extensively by our group, typically to investigate the behavior of two-dimensional quantum dots [34]. This is modified here since the devices now being considered are fully three dimensional, and so there are two dimensions corresponding to the transverse direction instead of just one. Each transverse plane contains $N_{y} \times N_{z}$ grid points. Normally, this would produce a second-rank tensor (matrix) for the wave function, and it would propagate via a fourthrank tensor. However, we can re-order the coefficients into a $N_{y} N_{z} \times 1$ first-rank tensor (i.e. a vector), so that the propagation is handled by a simpler matrix multiplication. Since the smallest dimension in our calculations is generally in the $z$ direction, we use $N_{z}$ for the expansion, and write the vector wave function as

$\Psi^{(i)}=\left[\begin{array}{c}\psi_{1,1}^{(i)} \\ \psi_{2,1}^{(i)} \\ \cdots \\ \psi_{N_{z}, N_{y}}^{(i)}\end{array}\right]$

Now, (2) can be rewritten as a matrix equation

$$
\begin{aligned}
& H^{(i)} \Psi^{(i)}(s)-T_{x}^{(i)} \Psi^{(i)}(s-1)-T_{x}^{(i)} \Psi^{(i)}(s+1) \\
& \quad=E I \Psi^{(i)}(s) .
\end{aligned}
$$

Here, $I$ is the unit matrix, $E$ is the energy to be found from the eigenvalue equation, while

$H^{(i)}=\left[\begin{array}{cccc}H_{0}^{(i)} & \tilde{t}_{z}^{(i)} & \ldots & 0 \\ \tilde{t}_{z}^{(i)} & H_{0}^{(i)}(\mathbf{r}) & \ldots & \ldots \\ \ldots & \ldots & \ldots & \tilde{t}_{z}^{(i)} \\ 0 & \ldots & \tilde{t}_{z}^{(i)} & H_{0}^{(i)}(\mathbf{r})\end{array}\right]$,

is a Hamiltonian corresponding to an individual slice, and

$T_{x}^{(i)}=\left[\begin{array}{cccc}\tilde{t}_{x}^{(i)} & 0 & \ldots & 0 \\ 0 & \tilde{t}_{x}^{(i)} & \ldots & 0 \\ \ldots & \ldots & \ldots & \ldots \\ 0 & 0 & \ldots & \tilde{t}_{x}^{(i)}\end{array}\right]$

represents the inter-slice coupling. The dimension of these two super-matrices is $N_{z} \times N_{z}$, while the basic $H_{0}$ terms of (7) have dimension of $N_{y} \times N_{y}$, so that the total dimension of the above two matrices is $N_{y} N_{z} \times N_{y} N_{z}$. In general, if we take $k$ and $j$ as indices along $y$, and $\eta$ and $v$ as indices along $z$, then

$\left(\tilde{t}_{z}^{(i)}\right)_{\eta \nu}=t_{z}^{(i)} \delta_{\eta \nu}, \quad\left(\tilde{t}_{y}^{(i)}\right)_{k j}=t_{y}^{(i)} \delta_{k j}$,

$\left(\tilde{t}_{x}^{(i)}\right)_{s s^{\prime}}=t_{z}^{(i)} \delta_{s s^{\prime}}$ 
and

$$
\begin{aligned}
& H_{0}^{(i)}(\mathbf{r}) \\
& =\left[\begin{array}{cccc}
V(s, 1, \eta)+W & t_{y}^{(i)} & \ldots & 0 \\
t_{y}^{(i)} & V(s, 2, \eta)+W & \ldots & 0 \\
\ldots & \ldots & \ldots & t_{y}^{(i)} \\
0 & 0 & t_{z}^{(i)} & V\left(s, N_{y}, \eta\right)+W
\end{array}\right] .
\end{aligned}
$$

Using (6) and the trivial equation $\psi_{s}^{(i)}=\psi_{s}^{(i)}$, one can construct a transfer matrix equation that relates adjacent pairs of slices:

$\left[\begin{array}{c}\psi_{s}^{(i)} \\ \psi_{s+1}^{(i)}\end{array}\right]=T_{s}\left[\begin{array}{c}\psi_{s-1}^{(i)} \\ \psi_{s}^{(i)}\end{array}\right]$,

where

$T_{s}=\left[\begin{array}{cc}0 & -I \\ -I & \left(H^{(i)}-E\right)\left(T_{x}^{(i)}\right)^{-1}\end{array}\right]$.

One begins by solving the eigenvalue problem on slice $s=0$ at the source end (away from the channel), which determines the propagating and evanescent modes for a given Fermi energy in this region. The eigenvectors of (11) have the general form

$\left[\begin{array}{c}\overrightarrow{u_{m}}( \pm) \\ \lambda_{m}( \pm) \overrightarrow{u_{m}}( \pm)\end{array}\right]$

Assuming there are $q$ propagating wave modes $\left(\left|\lambda_{m}\right|=1\right)$ in the source of the device and $N-q$ evanescent modes $\left(N=N_{y} N_{z}\right)$, the eigenvalues may be expressed as

$\lambda_{m}( \pm)=e^{ \pm i k_{m} a}, \quad m=1, \ldots, q$,

$\lambda_{m}( \pm)=e^{ \pm i \kappa_{m} a}, \quad m=q+1, \ldots, N$.

In the above equation, the \pm refers to the fact that the modes come in pairs of left $(-)$ and right $(+)$ traveling waves. One then collects these together into a matrix

$T_{\text {left }}=\left[\begin{array}{cc}U_{+}(0) & U_{-}(0) \\ \lambda_{+} U_{+}(0) & \lambda_{-} U_{-}(0)\end{array}\right]$,

where the matrices

$U_{ \pm}(0)=\left\lfloor u_{1}( \pm)(0) \cdots u_{N}( \pm)(0)\right\rfloor$

contain the mode eigenvectors for the $s=0$ slice, with the corresponding eigenvalue matrices

$\lambda_{ \pm}=\operatorname{diag}\left\lfloor\lambda_{1} \cdots \lambda_{N}( \pm)\right\rfloor$.

One also constructs a matrix for the drain end, $T_{\text {right }}$, which is obtained in exactly the same fashion, except that the calculation is done at $s=N_{z}$.
We now proceed to calculate the transmission from the source to the drain of the structure, which is done by solving the transfer matrix problem

$\left[\begin{array}{c}t \\ 0\end{array}\right]=T_{\text {right }}^{-1} T_{N} T_{N-1} \cdots T_{1} T_{\text {left }}\left[\begin{array}{l}I \\ r\end{array}\right]$,

where $t$ matrix of transmission amplitudes of waves exiting from the right part of the structure, and $r$ is the corresponding matrix of amplitudes of waves reflected back towards the left. The unit matrix, $I$, and the zero matrix, 0 , set the transport boundary conditions mentioned above. Given the matrix elements of $t$, one can calculate the total transmission for valley $i$ as a function of energy:

$T^{(i)}(E)=\sum_{m, n} \frac{v_{n}}{v_{m}}\left|t_{n, m}\right|^{2}$,

where $t_{n, m}$ represents the transmission amplitude of mode $n$ to mode $m$ and the summation is only over propagating modes. Here $v_{n}$ represents the velocity in the $x$-direction of $n$th mode, which can be obtained by taking the expectation value for each mode of the probability current operator in the $x$-direction expressed in the finite difference representation. The process must be repeated for each valley and the net transmission, $T(E)$ is the sum over all their contributions. Importantly, as outlined, this procedure assumes that each valley can be treated independently.

Unfortunately, the transfer matrix method is numerically unstable due to the exponentially growing and decaying contributions made by the evanescent modes. This problem can be overcome by replacing (17) with the following numerical stable procedure $[33,34]$, which essentially corresponds to a cascade of scattering matrices:

$$
\begin{aligned}
& {\left[\begin{array}{cc}
C_{1}^{(i)}(s+1) & C_{2}^{(i)}(s+1) \\
0 & 1
\end{array}\right]} \\
& \quad=T_{s}\left[\begin{array}{cc}
C_{1}^{(i)}(s) & C_{2}^{(i)}(s) \\
0 & 1
\end{array}\right]\left[\begin{array}{cc}
1 & 0 \\
P_{1}^{(i)}(s) & P_{2}^{(i)}(s)
\end{array}\right] .
\end{aligned}
$$

The dimension of these matrices is $2 N_{y} N_{z} \times 2 N_{y} N_{z}$, but the effective propagation is handled by submatrix computations, through the fact that the second row of this equation sets the iteration conditions

$$
\begin{aligned}
C_{2}^{(i)}(s+1) & =P_{2}^{(i)}(s) \\
& =\left[-C_{2}^{(i)}(s)+\left(T_{x}^{(i)}\right)^{-1}\left(E I-H^{(i)}\right)\right]^{-1}, \\
C_{1}^{(i)}(s+1) & =P_{1}^{(i)}(s)=P_{2}^{(i)}(s) C_{1}^{(i)}(s) .
\end{aligned}
$$

Under this modified procedure, $C_{1}(0)=1$, and $C_{2}(0)=0$ are used as the initial conditions at the source end. These are now propagated to the $N_{x}$ slice, which is the end of the 
active region. The final transmission matrix is then obtained from:

$$
\begin{aligned}
t= & -\left(U_{+}\left(N_{x}\right) \lambda_{+}\left(N_{x}\right)\right)^{-1} \\
& \times\left[C_{1}^{(i)}\left(N_{x}+1\right)-U_{+}\left(N_{x}\right)\left(U_{+}\left(N_{x}\right) \lambda_{+}\left(N_{x}\right)\right)^{-1}\right]^{-1} .
\end{aligned}
$$

It should be noted that (20) is a form of the Dyson's equation for iterative solutions of Green's function type behavior [35]. In fact, the two $C$ parameters are proper Green's functions, but are normalized by the hopping terms to be dimensionless. Here, $C_{2}^{(i)}$ plays the role of the onsite Green's function $G(i, i)$, and $C_{1}^{(i)}$ plays the role of the on-site Green's function $G(0, i)$ [29]. Hence, the hopping Hamiltonian already plays the role of the hopping selfenergy term that couples one slice to the next.

Given the transmission, one can calculate the current flowing through the device for a given source-drain bias, $V_{s d}$ :

$I\left(V_{s d}\right)=\frac{2 e}{h} \int d E \cdot T(E)\left[f_{s}(E)-f_{d}(E)\right]$.

In the above equation, $f_{s}$ and $f_{d}$ are the values of the Fermi functions at the source contact and drain contacts, respectively, and must be evaluated with the appropriate temperatures and voltages.

If we are to incorporate a self-consistent potential within the device, we must also solve Poisson's equation and for that the electron density is required. Usuki et al. outlined a method [33] for obtaining the density starting from the left and working back to the end of the structure. Unfortunately, it entails performing a calculation similar to that for obtaining the transmission, but for every single slice. As a result, while the time it takes to calculate $T$ goes as $N_{x}$, the time to reconstruct the wave function instead goes as $N_{x}$ !, which makes it very time consuming. Since self-consistency requires the density to be recalculated numerous times, including it becomes impractical for most calculations. We however found a simple way to make the reconstruction far more efficient. Instead of going from left to right, one starts at the end of the structure and works backword. Manipulating Usuki et al.'s equations, it can be shown [34] that for the final slice:

$\psi_{\xi}^{\left(N_{x}+1, i\right)}(j, \eta)=P_{1}^{(i)}\left(N_{x}+1\right)$.

Here, as before, the superscript $i$ denotes the valley, while $j$ and $\eta$ denote the transverse position and $\xi$ is the mode index. One can then proceed to the source end by using the following recursion relation:

$\psi_{\xi}^{(s, i)}(j, \eta)=P_{1}^{(i)}(s)+P_{2}^{(i)}(s) \psi_{\xi}^{(s+1, i)}(j, \eta)$.

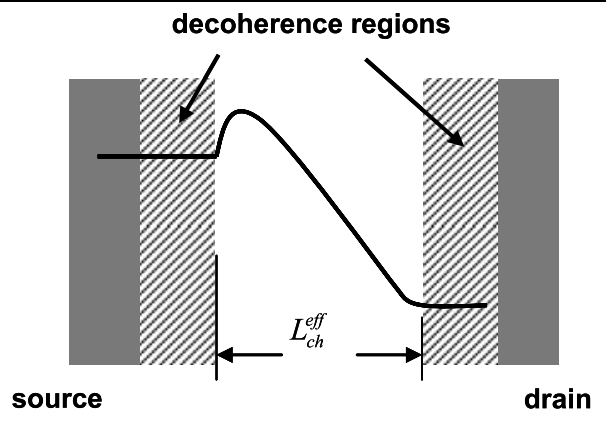

Fig. 2 A conceptual picture of a MOSFET under bias. The source and drain, as indicated by the two gray areas, may be considered to be the "contacts." The areas to the left and right of the effective channel length indicated here as the decoherence regions, must be considered part of the active device

Finally, the probability density at any site $(s, j, \eta)$ is found by taking the sum over $\xi$ of the occupied modes at that site, as

$n_{L}^{(i)}(s, j, \eta)=\sum_{\xi}\left|\psi_{\xi}^{(s, i)}(j, \eta)\right|^{2}$.

This expression gives the contribution to the probability density from valley $i$ assuming that the electron waves are incident from the left. Importantly, when one solves the Poisson equation, one must also compute and add in the contribution to the density from waves that start from the right, which means repeating the transmission and density calculation outlined above but translating from drain to source. To understand why this is so, consider the simplified potential profile of a MOSFET under bias shown in Fig. 2. From the source end, there is a small potential barrier between the source and the channel, and then the potential falls to the level of the drain potential (the energy is shown, this has a negative sign from the voltage). In his elementary 1D theory of ballistic transport, Lundstrom [36] identifies two major scattering regions, the barrier between the channel and the source, and within the channel, each of which contributes separate reflection coefficients. Higher reflectance of course means lower transmission and thus lower current. As one might expect, the shape of the potential barrier is of key importance with respect to determining just how much transmission there is. The nature of the barrier in Fig. 2 is that of a self-consistent potential subject to a constraint of the applied gate and drain voltages. The exact distribution of charge in the channel and in the drain will affect this potential barrier due to the nonlinear feedback of solving Poisson's equation. Thus, one needs the contribution to the total density starting from the drain end, as well as from the source in order for this to be accurately modeled.

Before being fed into the 3D Poisson equation, the probability density is then weighted by the appropriate thermal and density of states factors [37-39] and converted into a 
true electron density. In our calculations, one starts from an initial potential profile and solves the transport problem to obtain the density. A new potential profile is then obtained from the density by solving Poisson's equation and then the transport problem is solved again. This process repeated until a desired level of convergence is obtained.

\section{Treatment of scattering by the inclusion of a self-energy term}

Because of the form of equation (20), it becomes quite simple to modify the recursive formulation by the addition of an on-site self-energy:

$$
H_{0} \equiv H^{(i)}(s) \rightarrow H=H^{(i)}(s)+\Sigma_{i, s} .
$$

The self-energy $\Sigma$ has both real and imaginary parts, with the latter representing the dissipative interactions. In semiconductors, the scattering is weak, and is traditionally treated by first-order time-dependent perturbation theory, which yields the common Fermi golden rule for scattering rates. With such weak scattering, the real part of the selfenergy can generally be ignored for the phonon interactions, and that part that arises from the carrier-carrier interactions is incorporated into the solutions of Poisson's equation by a local-density approximation, which approximately accounts for the Hartree-Fock corrections [38]. In the many-body formulations of the self-energy, the latter is a two-site function in that it is written as [40]

$\Sigma\left(\mathbf{r}_{1}, \mathbf{r}_{2}\right)$

In our case, where we are using transverse modes in the quantum wire, this may be rewritten as

$$
\Sigma\left(i, j ; i^{\prime}, j^{\prime}, x_{1}, x_{2}\right) .
$$

Here, the scattering accounts for transitions from transverse mode $i, j$ at position $x_{1}$ to $i^{\prime}, j^{\prime}$ at position $x_{2}$. Generally, one then makes a center-of-mass transformation [40, 41]

$X=\frac{x_{1}+x_{2}}{2}, \quad \xi=x_{1}-x_{2}$,

and then Fourier transforms on the difference variable to give

$$
\begin{aligned}
& \Sigma\left(i, j ; i^{\prime}, j^{\prime}, X, k_{x}\right) \\
& \quad=\frac{1}{2 \pi} \int d \xi e^{i \xi k_{x}} \Sigma\left(i, j ; i^{\prime}, j^{\prime}, X, \xi\right) .
\end{aligned}
$$

The center-of-mass position $X$ remains in the problem as the mode structure may change as one moves along the channel. At this point, the left-hand side of (30) is the self-energy computed by the normal scattering rates, such as is done in quantum wells and quantum wires previously [30, 31]. However, these previous calculation usually used the Fermi golden rule, which is an evaluation of the bare self-energy in (30). In many-body approaches, one normally does not use the energy-conserving delta function that is the central part of the Fermi golden rule. Rather, this function is broadened into the spectral density, through the use of the selfconsistent Born approximation (see, e.g. [9]). In this way, off shell effects are taken into account through this broadening of the relationship between momentum and energy. In semiconductors, however, we have already noted that the scattering is weak. It has been pointed out that these offshell corrections are only important in fast processes where we are interested in femtosecond response and their neglect introduces only slight errors for times large compared to the collision duration [42]. Moreover, the broadening of the delta function will not be apparent when we reverse the Fourier transform of (30), as the area under the spectral density remains normalized to unity [38]. Since our recursion in (20) is in the site representation, rather than in a mode representation, we have to reverse the Fourier transform in (30) to get the $x$-axis variation, and do a mode-to-site unitary transformation to get the self-energy in the form necessary for the recursion. This is the subject of the rest of this section, where we discuss the different phonon scattering processes. Hence, we begin by seeking the imaginary part of the self-energy, which is related to the scattering rate via

$\operatorname{Im}\left\{\Sigma\left(i, j ; i^{\prime}, j^{\prime}, X, k_{x}\right)\right\}=\hbar\left(\frac{1}{\tau}\right)_{i, j}^{i^{\prime}, j^{\prime}}$.

It is the latter scattering rate which we calculate for the various scattering processes. This result will be a function of the $x$-directed momentum (which is related, in turn, to the energy of the carrier) in the quantum wire. This scattering rate must be converted to the site representation with a unitary transformation

$\Gamma=\operatorname{Im}\{\Sigma\}=U^{+}\left(\frac{\hbar}{\tau}\right)_{i, j}^{i^{\prime}, j^{\prime}} U$,

where $U$ is a unitary mode-to-site transformation matrix as discussed in Sect. 2. The unitary matrix $U^{+}$results from the eigenvector solutions in the transverse slice and are composed of the various eigenfunctions in the site basis. Hence, it represents a mode-to-slice transformation.

Besides the corrections to the site energies as outlined above, scattering introduces another complication if intervalley processes are allowed. The transmission method as described in Sect. 2 assumes that there is no coupling between valleys, and the calculation is done one valley at a 


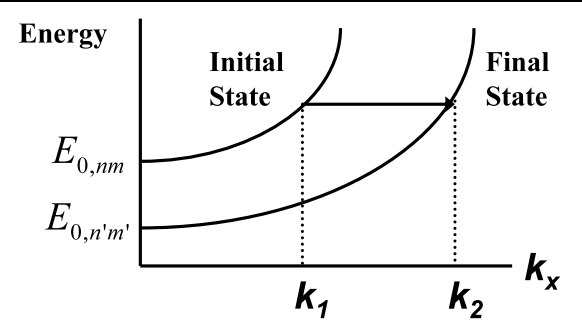

Fig. 3 Schematic of the parabolic bandstructure used in the formulation of the scattering method

time. When inter-valley scattering is allowed, then the contributions from valleys must be treated in tandem. It is the same basic calculation, except that the matrices are all expanded in size and new off-diagonal terms appear. For example, $U$ and $U^{+}$must now contain eigenvectors from all the valleys, not just one.

We now consider the case of acoustic phonon scattering. The Fermi golden rule scattering rate for acoustic phonons is treated in nearly all textbooks, and the only modification is to account for the transverse modes of the quantum wire. As mentioned above, this has previously been treated for quantum wells and for silicon quantum wires. Hence, we begin with the general form

$$
\left(\frac{1}{\tau}\right)_{i, j}^{i^{\prime}, j^{\prime}}=\frac{2 \pi}{\hbar} \frac{D_{a c}^{2} k_{B} T}{2 \rho v_{s}^{2}} I_{n^{\prime} m^{\prime}}^{n m} \sum_{\vec{k}^{\prime}} \delta\left(E_{k^{\prime}}-E_{k}\right) .
$$

Here, the acoustic phonon is treated, as is normal, as quasielastic in that the energy transferred to the acoustic mode is considerably smaller than the carrier energy [43, 44], and the delta function in (9) serves to conserve the energy in the process. The overlap integral gives the mode-to-mode coupling, as

$I_{i, j}^{i^{\prime}, j^{\prime}}=\frac{A}{4 \pi^{2} V} \iint d y d z\left[\varphi_{i, j}^{*}(y, z) \varphi_{i^{\prime}, j^{\prime}}(y, z)\right]^{2}$,

where $\varphi_{i, j}(y, z)$ is the transverse wave function in the particular slice. The difference here from the form in a quantum well is that these transverse wave functions are twodimensional rather than one-dimensional, while the momentum vector $k$ is a one-dimensional quantity.

In the above equations, $E_{k}$ and $E_{k^{\prime}}$ are the energies corresponding to the initial and final energy states in the assumed parabolic subbands, which may be defined through the use of Fig. 3. Here, we illustrate a simple two subband model to define the initial and final energies. $E_{0, i j}$ is the energy value corresponding to $k_{x}=0$ in the initial subband, while $E_{0, i^{\prime} j^{\prime}}$ corresponds to the value of the energy in the final subband with a $k_{x}^{\prime}=0$ value. With these definitions,

$E_{k}=E_{0, i j}+\frac{\hbar^{2} k_{1}^{2}}{2 m_{x}^{*}}$,

and

$E_{k^{\prime}}=E_{0, i^{\prime} j^{\prime}}+\frac{\hbar^{2} k_{2}^{2}}{2 m_{x}^{*}}$.

From this, the difference between the initial and final energies becomes

$E_{k}-E_{k^{\prime}}=E_{0, i j}-E_{0, i^{\prime} j^{\prime}}+\frac{\hbar^{2}}{2 m_{x}^{*}}\left(k_{1}^{2}-k_{2}^{2}\right)$.

To solve for $k_{2}$ in terms of $k_{1}$ and the difference between the initial and final energies, we denote

$\Delta_{i j}^{i^{\prime} j^{\prime}}=E_{0, i j}-E_{0, i^{\prime} j^{\prime}}$

and

$k_{2}^{2}=k_{1}^{2}+\frac{2 m_{x}^{*}}{\hbar^{2}} \Delta_{i j}^{i^{\prime} j^{\prime}}$.

Following the usual procedure, the summation over final momentum states is replaced with an integration, as

$\sum_{k^{\prime}} \rightarrow \frac{L}{2 \pi} \int_{-\infty}^{\infty} d k^{\prime}=\int_{0}^{\infty} \rho_{1 D}\left(E^{\prime}\right) d E^{\prime}$.

We now combine (40) with (34) to obtain

$$
\left(\frac{1}{\tau_{a c}}\right)_{i, j}^{i^{\prime}, j^{\prime}}=\frac{\pi D_{a c}^{2} k_{B} T}{\hbar \rho v_{s}^{2}} I_{i, j}^{i^{\prime}, j^{\prime}} \frac{L}{2 \pi} \frac{1}{\left|\frac{\partial E_{k^{\prime}}}{\partial k^{\prime}}\right|},
$$

where the last term is evaluated using (39). The scattering rate is then

$$
\begin{aligned}
& \left(\frac{1}{\tau_{a c}}\right)_{i, j}^{i^{\prime}, j^{\prime}} \\
& \quad=\frac{m_{x}^{*} D_{a c}^{2} k_{B} T}{2 \hbar^{3} \rho v_{s}^{2}} \frac{L I_{i, j}^{i^{\prime}, j^{\prime}}}{\sqrt{k^{2}+\frac{2 m_{x}^{*} \Delta_{i j}^{i^{\prime} j^{\prime}}}{\hbar^{2}}}} \theta\left(k^{2}+\frac{2 m_{x}^{*} \Delta_{i j}^{i^{\prime} j^{\prime}}}{\hbar^{2}}\right),
\end{aligned}
$$

where $\theta$ is the Heavyside step function $[\theta(x)=1$ for $x>0$, and 0 for $x<0$ ].

Now, for our real space quantum transport approach, we need to reverse the Fourier transform in (30). That is, we use the inverse transform to real space from momentum space and obtain the final form for the acoustic deformation potential scattering rate. The Fourier integral is 


$$
\begin{aligned}
& \left(\frac{1}{\tau}\right)_{i, j}^{i^{\prime}, j^{\prime}}\left(x-x^{\prime}\right) \\
& \quad=\frac{m_{x}^{*} D_{a c}^{2} k_{B} T}{2 \hbar^{3} \rho v_{s}^{2}}\left(L I_{i, j}^{i^{\prime}, j^{\prime}}\right) \frac{1}{\sqrt{2 \pi}} \int_{\beta}^{\infty} \frac{e^{i k\left(x-x^{\prime}\right)}}{\sqrt{k^{2}+\frac{2 m_{x}^{*} \Delta_{i j}^{i^{\prime} j^{\prime}}}{\hbar^{2}}}} d k,
\end{aligned}
$$

$\beta=\sqrt{-\frac{2 m_{x}^{*} \Delta_{i j}^{i^{\prime} j^{\prime}}}{\hbar^{2}}}$.

The lower limit in the integration results in zero if $\Delta_{i j}^{i^{\prime} j^{\prime}}>0$. From Fig. 2, it can be seen that scattering cannot occur from the lower subband to the upper subband unless there is a minimum momentum (or energy), and this accounts for the non-zero lower limit in the integration for such situations. We consider the case of $\Delta_{i j}^{i^{\prime} j^{\prime}} \leq 0$ here, and the integration can then be carried out easily to yield (the other cases are also easily done)

$$
\begin{aligned}
\left(\frac{1}{\tau}\right)_{i, j}^{i^{\prime}, j^{\prime}}\left(x-x^{\prime}\right) & \\
= & \frac{m_{x}^{*} D_{a c}^{2} k_{B} T}{2 \hbar^{3} \rho v_{s}^{2}}\left(L I_{i, j}^{i^{\prime}, j^{\prime}}\right) \frac{1}{\sqrt{2 \pi}} \\
& \times\left\{\frac{\pi}{2}-\beta \operatorname{Si}\left[-i \beta\left(x-x^{\prime}\right)\right] \cosh \left[\beta\left(x-x^{\prime}\right)\right]\right. \\
& \left.-\beta C i\left[-i \beta\left(x-x^{\prime}\right)\right] \sinh \left[\beta\left(x-x^{\prime}\right)\right]\right\} .
\end{aligned}
$$

The term in curly brackets is sharply peaked around $x=$ $x^{\prime}$, which implies the scattering is local with regard to the individual slices in the recursion. There is coupling between the modes within a slice, but this local (to the slice) behavior is just the normal assumption in quasi-classical cases, where the scattering is assumed to be local in space [45]. Yet we need to know the total scattering rate within the slice, so this is achieved by integrating over $x^{\prime}$ in order to find the resultant scattering weight

$$
\left(\frac{1}{\tau_{a c}}\right)_{i, j}^{i^{\prime}, j^{\prime}}=\frac{m_{x}^{*} D_{a c}^{2} k_{B} T}{4 \hbar^{3} \rho v_{s}^{2}}\left(L I_{i, j}^{i^{\prime}, j^{\prime}}\right) \sqrt{\frac{\pi}{2}} .
$$

Finally, this scattering rate must be converted to the site representation with a unitary transformation given by (32).

Scattering between the equivalent valleys of silicon is carried out by high energy optical modes. In general, there two types of phonons, the $f$ phonons between valleys on different axes in momentum space and $g$ phonons between the two valleys along the same coordinate axis [46, 47]. There are several phonons which can contribute to each of these processes [48], but the treatment for each is the same. The differences from the acoustic mode treatment lie in the fact that the energy of the phonon can no longer be ignored in (37), and the phonon distribution function cannot be approximated by a equi-partition approximation. Hence, we need to treat the emission and absorption of phonons differently, but the result is very similar. In general, the case for phonon absorption yields the result (analogous to (42))

$$
\begin{aligned}
\left(\frac{1}{\tau_{i v \_a b}}\right)_{i, j}^{i^{\prime}, j^{\prime}}= & \frac{m_{x}^{*} D_{i v}^{2} N_{q}}{8 \pi^{2} \rho \hbar^{2} \omega_{q} L}\left(I_{i, j}^{i^{\prime}, j^{\prime}}\right) \\
& \times \frac{1}{\sqrt{k^{2}+\frac{2 m_{x}^{*} \Delta_{i, j}^{i^{\prime}, j^{\prime}}}{\hbar^{2}}}} \theta\left(k^{2}+\frac{2 m_{x}^{*} \Delta_{i, j}^{i^{\prime}, j^{\prime}}}{\hbar^{2}}\right),
\end{aligned}
$$

where now

$\Delta_{i j}^{i^{\prime} j^{\prime}}=E_{0, i j}-E_{0, i^{\prime} j^{\prime}}+\hbar \omega_{q}$.

In the case of the emission of a phonon, $N_{q}$ in (46) is replaced by $N_{q}+1$, and the positive sign in front of the phonon energy in (47) is replaced by a negative sign. However, there are further complications in the case of phonon emission which we will address below. For the absorption case, it is apparent that the form of the resulting equation is the same as that of the acoustic phonons, and we can use the same integration results for the inverse Fourier transform and for the site summation to yield the scattering strength. Basically, only the parameters in the leading coefficient are changed. Hence, we can immediately adapt (45) to give the result

$\left(\frac{1}{\tau_{i v \_a b}}\right)_{i, j}^{i^{\prime}, j^{\prime}}=\frac{m_{x}^{*} D_{i v}^{2} N_{q}}{8 \pi^{2} \rho \hbar^{2} \omega_{q} L}\left(I_{i, j}^{i^{\prime}, j^{\prime}}\right) \sqrt{\frac{\pi}{2}}$.

Once again, this is now inserted into (32) to get the site representation.

As mentioned above, the emission case has some complications. We must consider three different cases corresponding to different values for the energy difference from the initial subband to the final subband and the emitted phonon energy: $\Delta_{i j}^{i^{\prime} j^{\prime}}<0, \Delta_{i j}^{i^{\prime} j^{\prime}}>0$, and $\Delta_{i j}^{i^{\prime} j^{\prime}}=0$. In each case, the Fourier transform is solved through the use of contour integrations.

1. $\Delta_{i j}^{i^{\prime} j^{\prime}}<0$. The resultant Fourier integral is

$$
\begin{aligned}
\left(\frac{1}{\tau}\right)_{i, j}^{i^{\prime}, j^{\prime}}\left(x-x^{\prime}\right)= & \frac{m_{x}^{*} D_{i v}^{2}\left(N_{q}+1\right)}{8 \hbar^{2} \rho \omega_{q} L}\left(I_{i, j}^{i^{\prime}, j^{\prime}}\right) \\
& \times \frac{1}{\sqrt{2 \pi}} \int_{0}^{\infty} \frac{\cos \left(k_{0}\left(x-x^{\prime}\right)\right)}{\sqrt{k^{2}-\beta^{2}}} d k .
\end{aligned}
$$

While an exact closed form for the integral cannot be found, it can be closely approximated as 


$$
\begin{aligned}
\left(\frac{1}{\tau_{i v \_e m}}\right)_{i, j}^{i^{\prime}, j^{\prime}}\left(x-x^{\prime}\right)= & \frac{m_{x}^{*} D_{i v}^{2}\left(N_{q}+1\right)}{8 \pi^{2} \rho \hbar^{2} \omega_{q} L}\left(I_{i, j}^{i^{\prime}, j^{\prime}}\right) \\
& \times\left[-\frac{\pi}{2} N_{0}\left(x-x^{\prime}\right)\right] .
\end{aligned}
$$

As previously, this is localized, and we integrate over $x^{\prime}$ to get the total scattering strength. This leads to

$\left(\frac{1}{\tau_{i v \_e m}}\right)_{i, j}^{i^{\prime}, j^{\prime}}=\frac{m_{x}^{*} D_{i v}^{2}\left(N_{q}+1\right)}{8 \pi^{2} \rho \hbar^{2} \omega_{q} L}\left(I_{i, j}^{i^{\prime}, j^{\prime}}\right)$.

2. $\Delta_{i j}^{i^{\prime} j^{\prime}}>0$. The major factor here is the change in the sign of the second term in the square root in the denominator of (46), which leads to

$$
\begin{aligned}
\left(\frac{1}{\tau}\right)_{i, j}^{i^{\prime}, j^{\prime}}\left(x-x^{\prime}\right) \\
=\frac{m_{x}^{*} D_{i v}^{2}\left(N_{q}+1\right)}{8 \hbar^{2} \rho \omega_{q} L}\left(I_{i, j}^{i^{\prime}, j^{\prime}}\right) \\
\quad \times \frac{1}{\sqrt{2 \pi}} \int_{0}^{\infty} \frac{\cos \left(k_{0}\left(x-x^{\prime}\right)\right)}{\sqrt{k^{2}+\beta^{2}}} d k \\
=\frac{m_{x}^{*} D_{i v}^{2}\left(N_{q}+1\right)}{8 \hbar^{2} \rho \omega_{q} L}\left(I_{i, j}^{i^{\prime}, j^{\prime}}\right) \frac{1}{\sqrt{2 \pi}} K_{0}\left(x-x^{\prime}\right) .
\end{aligned}
$$

Using an asymptotic expansion of the Bessel function of an imaginary argument results in

$$
\begin{aligned}
\left(\frac{1}{\tau}\right)_{i, j}^{i^{\prime}, j^{\prime}}\left(x-x^{\prime}\right)= & \frac{m_{x}^{*} D_{i v}^{2}\left(N_{q}+1\right)}{8 \hbar^{2} \rho \omega_{q} L}\left(I_{i, j}^{i^{\prime}, j^{\prime}}\right) \\
& \times \frac{1}{\sqrt{2 \pi}} \sqrt{\frac{\pi}{2\left(x-x^{\prime}\right)}} e^{-\left(x-x^{\prime}\right)} .
\end{aligned}
$$

We now integrate $x^{\prime}$ to determine the total scattering strength. The final result is

$$
\left(\frac{1}{\tau_{i v \_e m}}\right)_{i, j}^{i^{\prime}, j^{\prime}}\left(x-x^{\prime}\right)=\frac{m_{x}^{*} D_{i v}^{2}\left(N_{q}+1\right)}{16 \pi \rho \hbar^{2} \omega_{q} L}\left(I_{i, j}^{i^{\prime}, j^{\prime}}\right) \Gamma\left(\frac{3}{2}\right) .
$$

3. $\Delta_{i j}^{i^{\prime} j^{\prime}}=0$. In this case, the Fourier integral is

$$
\begin{aligned}
\left(\frac{1}{\tau}\right)_{i, j}^{i^{\prime}, j^{\prime}}\left(x-x^{\prime}\right) & \\
= & \frac{m_{x}^{*} D_{i v}^{2}\left(N_{q}+1\right)}{8 \hbar^{2} \rho \omega_{q} L}\left(I_{i, j}^{i^{\prime}, j^{\prime}}\right) \\
& \times \frac{1}{\sqrt{2 \pi}} \int_{0}^{\infty} \frac{\cos \left(k_{0}\left(x-x^{\prime}\right)\right)}{k} d k \\
= & \frac{m_{x}^{*} D_{i v}^{2}\left(N_{q}+1\right)}{8 \hbar^{2} \rho \omega_{q} L}\left(I_{i, j}^{i^{\prime}, j^{\prime}}\right) \frac{1}{\sqrt{2 \pi}}\left[-C i\left(x-x^{\prime}\right)\right] .
\end{aligned}
$$

Once again, $x^{\prime}$ is integrated out of the equation to determine the magnitude of the delta function. The final result is

$$
\left(\frac{1}{\tau_{i v \_e m}}\right)_{n^{\prime} m^{\prime}}^{n m}=\frac{m_{x}^{*} D_{i v}^{2}\left(N_{q}+1\right)}{8 \sqrt{2} \pi^{\frac{5}{2}} \rho \hbar^{2} \omega_{q} L}\left(I_{n^{\prime} m^{\prime}}^{n m}\right)
$$

\section{Application of the method: determining the ballistic to diffusive crossover in a SOI MOSFET}

In this section, we show an application of the methodology described above. We consider an SOI MOSFET, with its channel aligned along the (110) direction, as shown earlier in Fig. 1. For the device under consideration here, the thickness of the silicon layer is $6.51 \mathrm{~nm}$. Oxide barriers were placed on either side of the channel to simulate the appearance of a hard wall boundary that would be present in an actual experimental system. The source and drain of the device are $36.93 \mathrm{~nm}$ wide and $27.15 \mathrm{~nm}$ in length. The source and drain of the device are discretely doped $n$-type with a doping concentration of $1 \times 10^{20} \mathrm{~cm}^{-3}$, while the channel is undoped. The quantum wire that forms the channel of the device has metal gates on three sides to form a trigate-type transistor [49]. The gate oxide thickness $\left(\mathrm{SiO}_{2}\right)$ on this device was $1 \mathrm{~nm}$. Figure 4 shows a cutaway view of our device, showing source, channel and drain, with dopant positions indicated. It should be noted that the effect of the presence of dopants in our approach is included as a local correction to the potential in the Hamiltonian. No scattering rate calculation is performed or required for them using our method. As would be the case in a real device, the dopants placed in random locations, according to the prescription outlined by Wong and Taur [50]. The same doping profile is used for

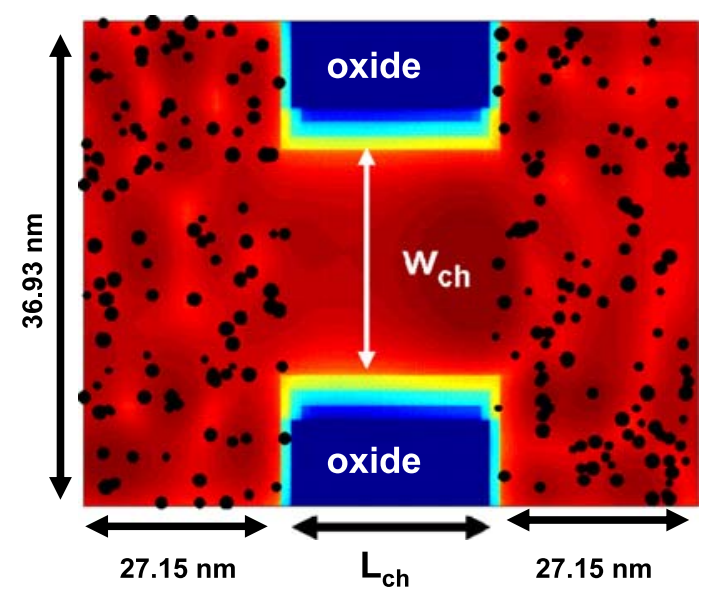

Fig. 4 Cutaway overview of the SOI MOSFET device, showing dopant atoms in the source and drain. The interior shading indicates the electron density. For clarity, a relatively long channel length was used to generate this picture 
each of the cases we examined, and the source and drain regions were left untouched at the channel length and channel width were varied. In every case, the gate voltage was set at $V_{g}=0.6 \mathrm{~V}$, while the source-drain bias was $V_{s d}=0.01 \mathrm{~V}$.

Our goal here is to determine the point where this MOSFET exhibits a ballistic to diffusive crossover as a function of channel length. In general, it has been suggested that the transport in small transistors is ballistic, and that once a carrier enters the channel it will continue to the drain, with no chance to scatter back to the source $[8,51]$. However, it has been shown that scattering within the channel will cause second-order effects which do affect the terminal characteristics of the transistor [52], and thus far, the search for ballistic behavior has not been so successful.

When the transport is ballistic, the resistance of the channel will be determined by the inverse of the Landauer conductance, as

$R_{\text {ballistic }}=\left[\frac{2 e^{2}}{h} N\right]^{-1}$,

where $N$ is the number of transverse modes propagating through the wire, $e$ is the electric charge and $h$ is Planck's constant. Importantly, there is no dependence upon the length of the wire. On the other hand, when the resistance is determined by the mobility $(\mu)$ and carrier density $(n)$, then the resistance is given by

$R_{\text {diffusive }}=\left[\frac{1}{\sigma}\right] \frac{L_{c h}}{A}=\frac{1}{n e \mu} \frac{L_{c h}}{A}$,

where $L_{c h}$ is the channel length and $A$ is the "cross-sectional area" of the inversion layer. We use the area here, rather than just the width of the two-dimensional layer, as we are dealing with a three-dimensional wire with full quantization in the transverse direction. Clearly, in this latter case, the resistance increases linearly with the channel length. It is the value of $L_{c h}$ where there is a change from (56) to (57) that we seek to determine.

Figure 5 shows the computed resistance for a device with a channel width of $6.5 \mathrm{~nm}$. For these results, acoustic deformation potential scattering and intervalley scattering with both $f$ and $g$ type processes were included. At a temperature of $100 \mathrm{~K}$, the electron-phonon scattering is largely suppressed, and in this case resistance quickly rises as function of channel length and then essentially saturates at $L_{c h} \sim 2 \mathrm{~nm}$. However, at $T=300 \mathrm{~K}$, the resistance continues to rise in a more or less linear fashion. This result is consistent with other simulations we have done with the channel aligned along the $\langle 001\rangle$ direction and at higher biases [26], which saw an apparent ballistic to diffusive crossover at $L_{c h} \sim 2 \mathrm{~nm}$. Note here that the resistance is actually lower for the $T=300 \mathrm{~K}$ case for very short channels lengths. In

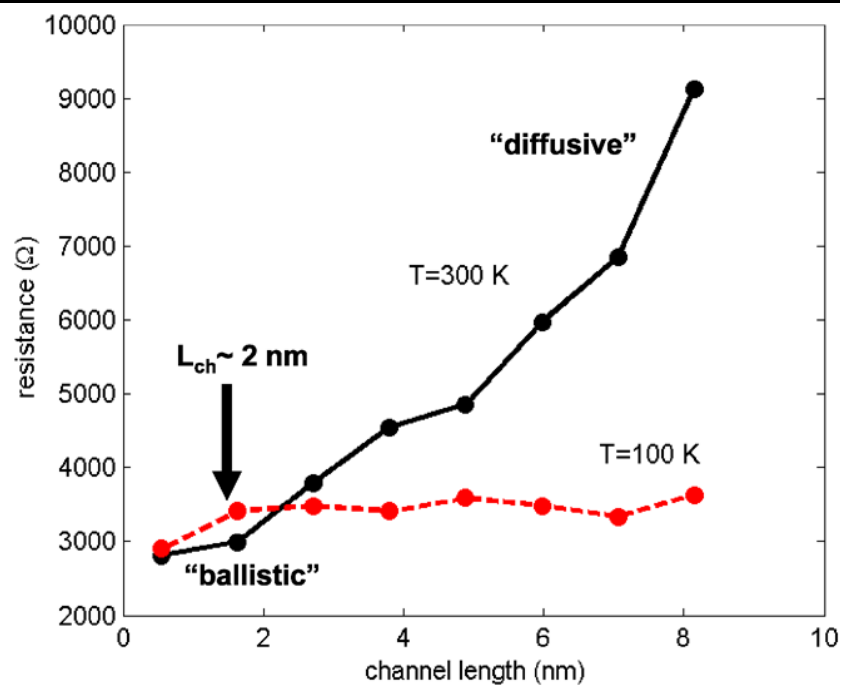

Fig. 5 Resistance vs. $L_{c h}$ for a device with channel width $w_{c h}=6.5 \mathrm{~nm}$ at $100 \mathrm{~K}$ and $300 \mathrm{~K}$

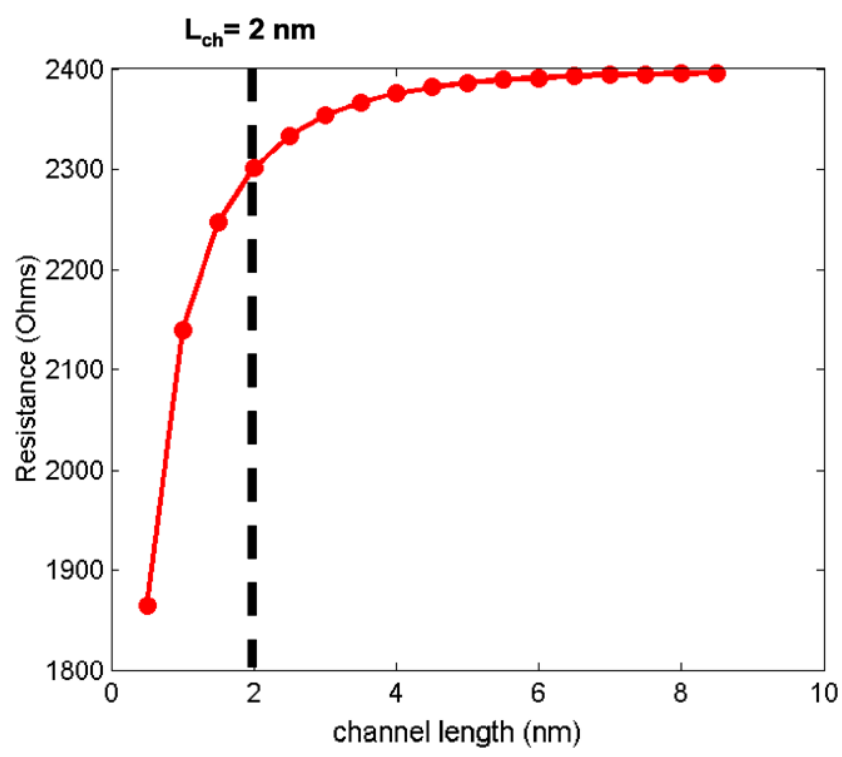

Fig. 6 Resistance vs. $L_{c h}$ for a device with channel width $w_{c h}=6.5 \mathrm{~nm}$ for the case of a perfect, ballistic wire without scattering

this particular case, conditions are such that the electronphonon scattering is able to excite electrons over the effective barrier created by the channel, thus reducing the resistance.

To understand what is special about $L_{c h} \sim 2 \mathrm{~nm}$, we have also performed a calculation for the ideal, ballistic case. This is shown in Fig. 6. Here, a perfect wire is used without any scattering at all from phonons or impurities, but with simple thermal broadening included using Fermi functions with $T=300 \mathrm{~K}$. As is evident, $L_{c h} \sim 2 \mathrm{~nm}$ is the approximate length for which the resistance saturates in the ballistic case. Beyond this length, the wire acts as an ideal quantum point contact (QPC) for which the transmission is quan- 

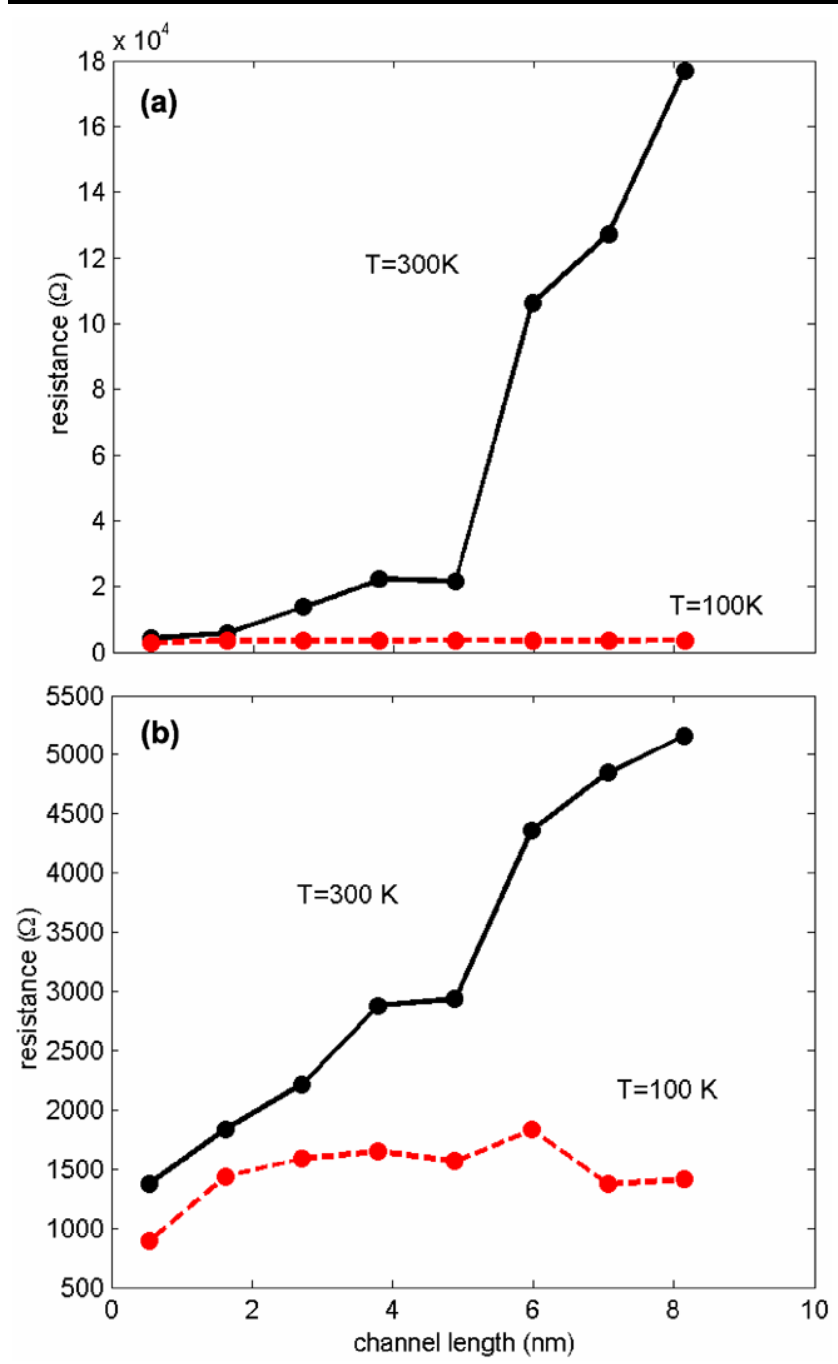

Fig. 7 (a) Resistance vs. $L_{c h}$ for a device with $w_{c h}=4.3 \mathrm{~nm}$ at the indicated temperatures. (b) As in (a), but for $w_{c h}=9.7 \mathrm{~nm}$

tized and proportional to the number of propagating modes in the wire. The effects of this quantization are evident even with the thermal broadening. However, at shorter lengths, evanescent wire modes that have not decayed can contribute, destroying the conductance quantization and decreasing the resistance.

Figures 7(a) and (b) shows results for a narrower $\left(w_{c h}=\right.$ $4.3 \mathrm{~nm})$ and a wider $\left(w_{c h}=9.7 \mathrm{~nm}\right)$ channel respectively. As in the first example, the $T=100 \mathrm{~K}$ and $T=300 \mathrm{~K}$ curves here appear to deviate in their behaviour at $L_{c h} \sim 2 \mathrm{~nm}$, indicating that once again that the effects of electron-phonon scattering start to dominate at that length. Both $T=300 \mathrm{~K}$ curves show a kink at $L_{c h} \sim 5 \mathrm{~nm}$ (this is also apparent in $w_{c h}=6.5 \mathrm{~nm}$ case, but is less pronounced). This effect is mainly due to the local impurity configuration at the ends of the channel happening to allow for a comparatively enhanced transmission in this particular case. Since we are dealing with discrete dopants that yield local poten-
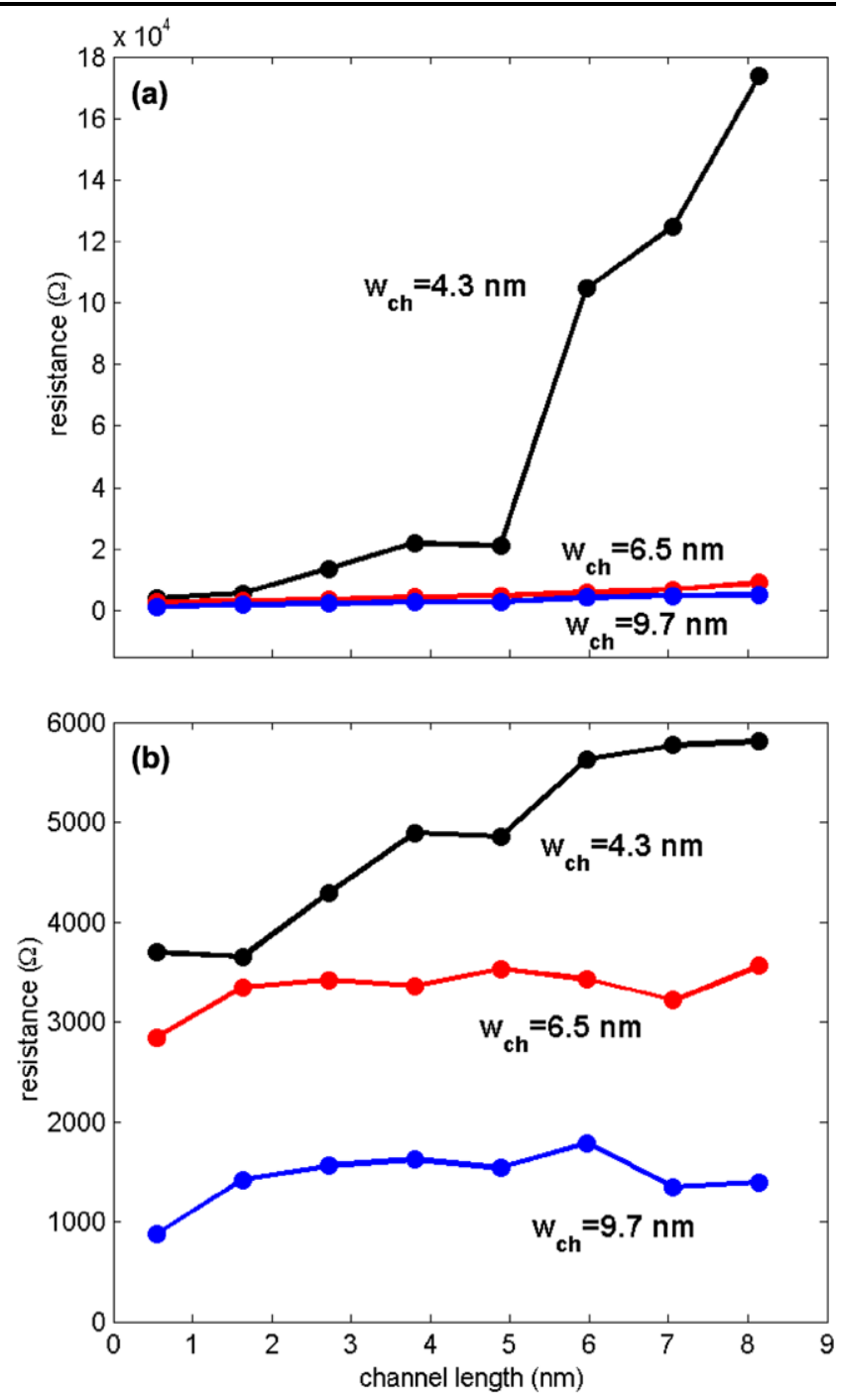

Fig. 8 (a) Direct comparison of the resistance results for the three channel width cases computed at $T=300 \mathrm{~K}$. (b) The same comparison, but for $T=100 \mathrm{~K}$

tial fluctuations that can either reflect or draw in electron waves, smooth curves will only occur if an ensemble average over different impurity configurations is performed. While the $w_{c h}=9.7 \mathrm{~nm}$ resistance looks approximately linear at $T=300 \mathrm{~K}$, the $w_{c h}=4.3 \mathrm{~nm}$ resistance appears to rise with a dependence that is more exponential in nature, as if what was being observed was akin to tunnelling. We note here that, at a width $w_{c h}=4.3 \mathrm{~nm}$, the wire is narrow enough to be near the point of pinch-off, where no modes are transmitted through the wire.

In Fig. 8, the results for the three channel widths are compared directly. Clearly, as shown in Fig. 8(a), the resistance in the $w_{c h}=4.3 \mathrm{~nm}$ case rises at a far more rapid rate at $300 \mathrm{~K}$ than for the wider channels. At $T=100 \mathrm{~K}$, the wider channels show the $L_{c h} \sim 2 \mathrm{~nm}$ saturation behaviour noted earlier, with a simple resistance offset separating the curves, 
as shown in Fig. 8(b). This is consistent with our discussion above about this length coinciding with the establishment of true QPC behavior. Interestingly, the $w_{c h}=4.3 \mathrm{~nm}$ resistance curve for $T=100 \mathrm{~K}$ looks somewhat like the $w_{c h}=9.7 \mathrm{~nm}$ result for $300 \mathrm{~K}$. Clearly, nearer to pinchoff, weaker electron-phonon scattering can have a more pronounced effect on the resistance.

\section{Conclusions}

In this paper, we have reviewed the technique that we have developed for performing quantum transport simulations of nanowire MOSFET transistors, which incorporates a simple physical approach to including separable scattering mechanisms as a self-energy correction in a real-space. Derivations of the scattering contributions for acoustic deformation potential and intervalley phonon scattering with both $f$ and $g$ type processes included were provided. Needless to say, any other scattering mechanisms that one chose to consider could be incorporated in a similar fashion. In that regard, it should be noted that our method is highly flexible and can be applied to many different kinds of systems. It has been used to simulate InAs tri-gate transistors [28], and transport through molecules [53], for example.

As an example application of our method, we presented results of transport simulations of short 3D silicon quantum wires including electron-phonon scattering, comparing results for different channel widths. At room temperature, we found that the resistance deviates from the ballistic prediction, even down to the length at which the wire becomes a true waveguide.

Acknowledgement This work was supported by the Office of Naval Research and by Intel Corporation.

\section{References}

1. Moore, G.E.: Electronics 38(8), 114 (1965)

2. Ramey, S.M., Ferry, D.K.: Physica B 314, 350 (2002)

3. Gardner, C.L., Ringhofer, C.: Phys. Rev. E 53, 157 (1996)

4. Ferry, D.K.: Superlattices Microstruct. 28, 419 (2000)

5. Asenov, A., Slavcheva, G., Brown, A.R., Davies, J.H., Saini, S.: IEEE Trans. Electron Devices 48, 722 (2001)

6. Ferry, K., Ramey, S.M., Shifren, L., Akis, R.: J. Comput. Electron. 1, 59 (2002)

7. Assad, F., Ren, Z., Vasileska, D., Datta, S., Lundstrom, M.: IEEE Trans. Electron Devices 47, 232 (2000)

8. Natori, K.: J. App. Phys. 76, 3730 (1994)

9. Lake, R., Klimeck, G., Bowen, R.C., Jovanovic, D.: J. App. Phys. 81, 7845 (1997)

10. Datta, S.: Superlattices Microstuct. 28, 253 (2000)
11. Knoch, J., Lengeler, B., Appenzeller, J.: IEEE Trans. Electron Devices 49, 1212 (2002)

12. Pikus, F.G., Likharev, K.K.: App. Phys. Lett. 71, 3661 (1997)

13. Laux, S.E., Kumar, A., Fischetti, M.V.: IEEE Trans. Nanotechnol. 1, 255 (2002)

14. Fishcetti, M.V.: J. App. Phys. 83, 270 (1988)

15. Benisty, H.: Phys. Rev. B 51, 13281 (1995)

16. Pala, M.G., Iannaccone, G.: Phys. Rev. Lett. 93, 256803 (2004)

17. Neofotistos, G., Lake, R., Datta, S.: Phys. Rev. B 43, 2442 (1991)

18. Akis, R., Bird, J.P., Ferry, D.K.: J. Phys. Condens. Matter 8, L667 (1996)

19. Frensley, W.R.: Rev. Mod. Phys. 62, 745 (1990)

20. Gebauer, R., Car, R.: Phys. Rev. B 70, 125324 (2004)

21. Büttiker, M.: Phys. Rev. Lett. 57, 1761 (1986)

22. Dekker, H.: Phys. Rev. A 16, 2126 (1977)

23. Kluksdahl, N.C., et al.: Phys. Rev. B 39, 7720 (1989)

24. Grubin, H.L., et al.: Semicond. Sci. Technol. 9, 855 (1994)

25. Gilbert, M., Ferry, D.K.: J. Appl. Phys. 95, 7954 (2004)

26. Gilbert, M.J., Akis, R., Ferry, D.K.: J. Appl. Phys. 98, 94303 (2005)

27. Akis, R., Gilbert, M., Ferry, D.K.: J. Phys. 38, 87 (2006)

28. Gilbert, M.J., Ferry, D.K.: J. Appl. Phys. 99, 054503 (2006)

29. Ferry, D.K., Goodnick, S.M.: Transport in Nanostructures. Cambridge University Press, Cambridge (1997)

30. Kotlyar, R., Obradovic, B., Matagne, P., Stettler, M., Giles, M.D.: Appl. Phys. Lett. 84, 5270 (2004)

31. Emberly, E.G., Kirczenow, G.: Phys. Rev. B 61, 5740 (2000)

32. Lake, R., Datta, S.: Phys. Rev. B 45, 6670 (1992)

33. Usuki, T., Saito, M., Takatsu, M., Kiehl, R.A., Yokoyama, N.: Phys. Rev. B 52, 8244 (1995)

34. Akis, R., Bird, J.P., Vasileska, D., Ferry, D.K., de Moura, A.P.S., Lai, Y.-C.: Electron Transport in Quantum Dots. Kluwer Academic, Dordrecht (2003). Chap. 6

35. Fetter, A.L., Walecka, J.D.: Quantum Theory of Many-Particle Systems. McGraw-Hill, New York (1971)

36. Lundstrom, M.: IEEE Electron Device Lett. 18, 361 (1997)

37. Sun, Y., Kirczenow, G.: Phys. Rev. B 47, 4413 (1993)

38. Wang, Y., Wang, J., Guo, H., Zaremba, E.: Phys. Rev. B 52, 2738 (1995)

39. Wang, C., Berggren, K.F.: Phys. Rev. B 54, 14257 (1996)

40. Kadanoff, L.P., Baym, G.: Quantum Statistical Mechanics. Benjamin/Cummings, Redwood City (1962)

41. Rammer, J.: Quantum Transport Theory. Perseus, Reading (1998)

42. Špička, V., Velický, B., Kalvová, A.: Physica E 29, 154 (2005)

43. Brooks, H.: Adv. Phys. 7, 85 (1955)

44. Schockley, W.: Electrons and Holes in Semiconductors. Van Nostrand, Princeton (1950)

45. Barker, J.R.: In: Ferry, D.K., Barker, J.R., Jacoboni, C. (eds.) Physics of Nonlinear Transport in Semiconductors, p. 126. Plenum Press, New York (1980)

46. Long, D.: Phys. Rev. 120, 2024 (1960)

47. Ferry, D.K.: Phys. Rev. B 12, 2361 (1975)

48. Ferry, D.K.: Semiconductors. Macmillan, New York (1991)

49. Doyle, H.S., Datta, S., Doczy, M., Hareland, S., Jin, B., Kavalieros, J., Linton, T., Murthy, A., Rios, R., Chau, R.: IEEE Electron Device Lett. 24, 263 (2003)

50. Wong, H.S., Taur, Y.: IEDM Tech. Dig., p. 705 (1993)

51. Lundstrom, M.: IEEE Electron Device Lett. 18, 361 (1997)

52. Svizhenko, A., Anantram, M.P.: IEEE Trans. Electron Devices 50, 1459 (2003)

53. Speyer, G., Akis, R., Ferry, D.K.: Superlattices Microstruct. 34, $429(2003)$ 